\title{
Shear bond strengths of seven self-etching primers after thermo-cycling
}

\section{Scherkräfte von sieben selbstätzenden Primern nach Thermozyklierung}

\author{
Lorenz Brauchli ${ }^{1}$, Marlis Zeller ${ }^{1}$, Andrea Wichelhaus ${ }^{2}$
}

\begin{abstract}
Objective. Self-etching primers are widely used in restorative dentistry. However, only one primer has been developed for orthodontic applications. The objective of this study was to assess the suitabiliy of restorative self-etching primers on unabraded enamel.

Material and Methods. Seven self-etching primers (Transbond $^{\mathrm{TM}} \mathrm{SEP}+$, Xeno $^{\circledR} \mathrm{III}$ Clearfil $^{\mathrm{TM}} \mathrm{SE}$, Futurabond ${ }^{\circledR} \mathrm{NR}$, One Coat, M-Bond and OptiBond ${ }^{\circledR}$ ) were compared with two conventionally-etched control groups (Transbond ${ }^{\mathrm{TM}} \mathrm{MIP}$ ). All groups with the exception of one of the two control groups underwent thermocycling $\left(1000 \mathrm{x}, 5-55^{\circ} \mathrm{C}\right)$. Bovine teeth stored in physiological saline solution were used for the purpose of this study.

Results. Both before and after thermo-cycling, the control groups presented very similar shear strengths which did not differ significantly from each other or from the Transbond ${ }^{\mathrm{TM}} \mathrm{SEP}+$ and Clearfil $^{\mathrm{TM}}$ SE specimens. During thermo-cycling, all groups except the control group and the Transbond ${ }^{\mathrm{TM}} \mathrm{SEP}+$ specimens displayed losses from the shear bodies. These losses occurred on all of the OptiBond $^{\circledR}$ samples. The adhesive remnant index (ARI) and enamel tear-outs correlated closely with the adhesive values.

Conclusions. Our adhesive value assessments support the conclusion that only Transbond ${ }^{\mathrm{TM}} \mathrm{SEP}+$ and $\mathrm{Clearfil}^{\mathrm{TM}} \mathrm{SE}$ can be recommended for clinical use.
\end{abstract}

\section{Keywords}

Shear strengths · Self-etching primers · Etching pattern

\section{Zusammenfassung}

Ziel. Selbstätzende Primer werden in der restaurativen Zahnheilkunde breit eingesetzt. Speziell für die kieferorthopädische Verwendung wurde allerdings nur ein einzelner Primer entwickelt. Ziel der vorliegenden Studie war es, die Eignung restaurativer selbstätzender Primer auf unbeschliffenem Schmelz zu untersuchen.

Material und Methodik. Sieben selbstätzende Primer (Transbond $^{\mathrm{TM}} \mathrm{SEP}+$, Xeno ${ }^{\mathrm{TM}} \mathrm{III}$, Clearfil ${ }^{\mathrm{TM}} \mathrm{SE}$, Futurabond NR, One Coat, M-Bond und OptiBond ${ }^{\circledR}$ ) wurden mit 2 konventionell geätzten Kontrollgruppen (Transbond ${ }^{\mathrm{TM}}$ MIP) verglichen. Alle Gruppen außer einer Kontrollgruppe wurden einer Thermozyklierung $\left(1000 x, 5-55^{\circ} \mathrm{C}\right)$ unterzogen. Für die Untersuchung wurden Rinderzähne verwendet, die in physiologischer Kochsalzlösung gelagert wurden.

Ergebnisse. Die Kontrollgruppen zeigten vor und nach Thermozyklierung sehr ähnliche Scherkräfte, die sich untereinander und gegenüber Transbond ${ }^{\mathrm{TM}} \mathrm{SEP}+$ und $^{\mathrm{Clearfil}}{ }^{\mathrm{TM}} \mathrm{SE}$ nicht signifikant unterschieden. Während der Thermozyklierung wurden für alle Gruppen außer der Kontrollgruppe und Transbond ${ }^{\mathrm{TM}}$ SEP+ Verluste der Scherkörper registriert. Bei OptiBond ${ }^{\circledR}$ fanden sich diese bei sämtlichen Proben. Der Adhäsiv-Rest-Index (ARI) sowie Schmelzausbrüche korrelierten gut mit den Haftwerten.

Schlussfolgerungen. Nur Transbond ${ }^{\mathrm{TM}} \mathrm{SEP}+$ und Clearfil $^{\mathrm{TM}} \mathrm{SE}$ können aufgrund der Haftwertuntersuchungen für den klinischen Einsatz empfohlen werden.

\section{Schlüsselwörter}

Scherkräfte · Selbstätzende Primer · Ätzmuster
${ }^{1}$ Clinic of Orthodontics and Pedodontics, University of Basel, Switzerland

${ }^{2}$ Department of Orthodontics, Ludwig-Maximilian University Munich, Germany

Received: January 17, 2011; accepted: July 15, 2011; published online: October 6, 2011
J Orofac Orthop 2011; 72:371-380

DOI 10.1007/s00056-011-0041-4 


\section{Introduction}

Adhesive technologies in dentisty and orthodontics have attracted serious interest since the introduction of acid etching by Buonocore [7] and bonding techniques introduced by Newman [33]. Since then, seven generations of adhesives have been described $[11,29]$. The first generation was the classic BIS-GMA, which remains a chief component of today's bonding systems; however, its main problem lay in its low adhesion to dentin. More hydrophile methacrylates were later developed which improved adhesion to dentin. It was not until the fourth generation that today's classic system encompassed hydrophilic primer, ambiphilic adhesive and hydrophobic bonding. After a renewed combination of the three substances in a single bottle system (fifth generation), the sixth generation delivered the self-etching primer. Most of these systems use a methacrylate-based phosphoric acid ester. With the seventh generation, an attempt was made to include anti-bacterial, anti-inflammatory and desensitizing substances; however, the effect of these substances is controversial $[11,25,36]$.

The main advantages of self-etching primers are that they reduce the likelihood of error and save time [15, 21, 49]. Their etching pattern is frequently less distinctive than with conventional phosphoric-acid etching [20,37, 42]. However, there is no proven correlation with adhesive forces [27]. This could be due to the identical depth of penetration of the methacrylate and etching acid when self-etching primers are used, something which cannot be guaranteed by conventional etching [13]. With self-etching primers, the etching process is stopped by dissolved calcium ions, increasing viscosity and ultimately by light-curing due to the inclusion of acid groups in the polymer matrix of the methacrylate [13]. Contact with oxygen causes incomplete hardening with active, residual acids on the surface of self-etching primers. These prevent combination with acid-sensitive, chemically-hardening resins. There is apparently no reduction in adhesion in conjunction with the combination with lightcuring products $[12,41]$. The bond between the self-etching primer and adhesive may weaken due to the accumulation of osmotically-active, dissolved hydroxyapatite in the unpolymerized surface layer $[46,47]$.

Transbond ${ }^{\mathrm{TM}}$ SEP+ (3M Unitek, Monrovia, CA, USA) was one of the first self-etching primers in orthodontics proven to be a suitable substitute for conventional etching methods in several clinical studies $[9,15,36,38]$. Yet some authors reported reduced adhesive values $[5,14,26,30]$. There is much less data on other self-etching primers, and their suitability for use on untreated enamel surfaces remains controversial [3, 6, 35, 42, 43].

In this study we examined the shear adhesion on unabraded enamel surfaces after thermo-cycling of the self-etching primers employed in conservative dentistry. Our objective was not primarily to re-examine the proven primer Transbond ${ }^{\mathrm{TM}} \mathrm{SEP}+$, but to establish the suitability of alternative primers for orthodontic applications.

\section{Einleitung}

Seit der Einführung der Säureätzung durch Buonocore [7] und der Klebetechniken in der Kieferorthopädie durch Newman [33] sind Adhäsivtechnologien für Zahnärzte und Kieferorthopäden von großem Interesse. Seither wurden 7 Generationen von Adhäsivstoffen beschrieben [11, 29]. Die erste Generation war das klassische BIS-GMA, das immer noch eine Hauptkomponente der heutigen Bondingsysteme darstellt. Das Hauptproblem lag in einer niedrigen Haftung auf Dentin. Nachfolgend wurden hydrophilere Methacrylate entwickelt, welche die Haftung auf Dentin verbessern konnten. Erst in der vierten Generation wurde das heute klassische System mit hydrophilem Primer, ambiphilem Adhäsiv und hydrophobem Bonding beschrieben. Nach erneuter Kombination der 3 Substanzen in einem Einflaschensystem (fünfte Generation), brachte die sechste Generation die selbstätzenden Primer. Die meisten Systeme verwenden dabei einen Phosphorsäureester auf Methacrylatbasis. In der siebten Generation wurde versucht, antibakterielle, antiinflammatorische und desensibilisierende Substanzen beizufügen. Diese sind in ihrer Wirkung jedoch umstritten $[11,25,36]$.

Der Hauptvorteil der selbstätzenden Primer besteht in der Reduktion der Fehlermöglichkeiten und einer Zeitersparnis $[15,21,49]$. Das Ätzmuster der selbstätzenden Primer ist oft schwächer ausgeprägt als bei konventioneller Phosphorsäureätzung [20,37, 42]. Allerdings wurde keine klare Korrelation zu den Haftkräften gefunden [27]. Dies könnte darauf beruhen, dass bei selbstätzenden Primern die Penetrationstiefe der Methacrylate mit jener der Ätzmittel identisch ist, was bei konventioneller Ätzung nicht gewährleistet werden kann [13]. Der Ätzprozess bei selbstätzenden Primern wird über herausgelöste Kalziumionen, eine zunehmende Viskosität und schließlich bei Lichthärtung durch den Einschluss der Säuregruppen in der Polymermatrix des Methacrylats gestoppt [13]. An der Oberfläche der selbstätzenden Primer entsteht durch den Kontakt mit Sauerstoff eine unvollständige Härtung mit weiterhin aktiven Säuregruppen. Diese verhindern die Kombination mit den säuresensitiven, chemisch härtenden Kunststoffen. Für die Kombination mit lichthärtenden Produkten fand sich keine Haftminderung [12, 41]. Eine Schwächung der Verbindung zwischen selbstätzendem Primer und verwendetem Adhäsiv könnte sich auch über eine Akkumulation von osmotisch aktivem, gelöstem Hydroxylapatit in der unpolymerisierten Oberflächenschicht ergeben $[46,47]$.

In der Kieferorthopädie erwies sich Transbond ${ }^{\mathrm{TM}} \mathrm{SEP}+(3 \mathrm{M}$ Unitek, Monrovia, CA, USA), einer der ersten selbstätzenden Primer, in mehreren klinischen Studien als geeigneter Ersatz für konventionelle Ätzmethoden $[9,15,36,38]$. Einige Autoren fanden aber auch verminderte Haftwerte [5, 14, 26, 30]. Für andere selbstätzende Primer bestehen deutlich weniger und kontroverse Daten zu deren Eignung auf unbehandelten Schmelzoberflächen $[3,6,35,42,43]$.

Die vorliegende Untersuchung prüfte selbstätzende Primer aus der konservierenden Zahnheilkunde bezüglich der Scher- 

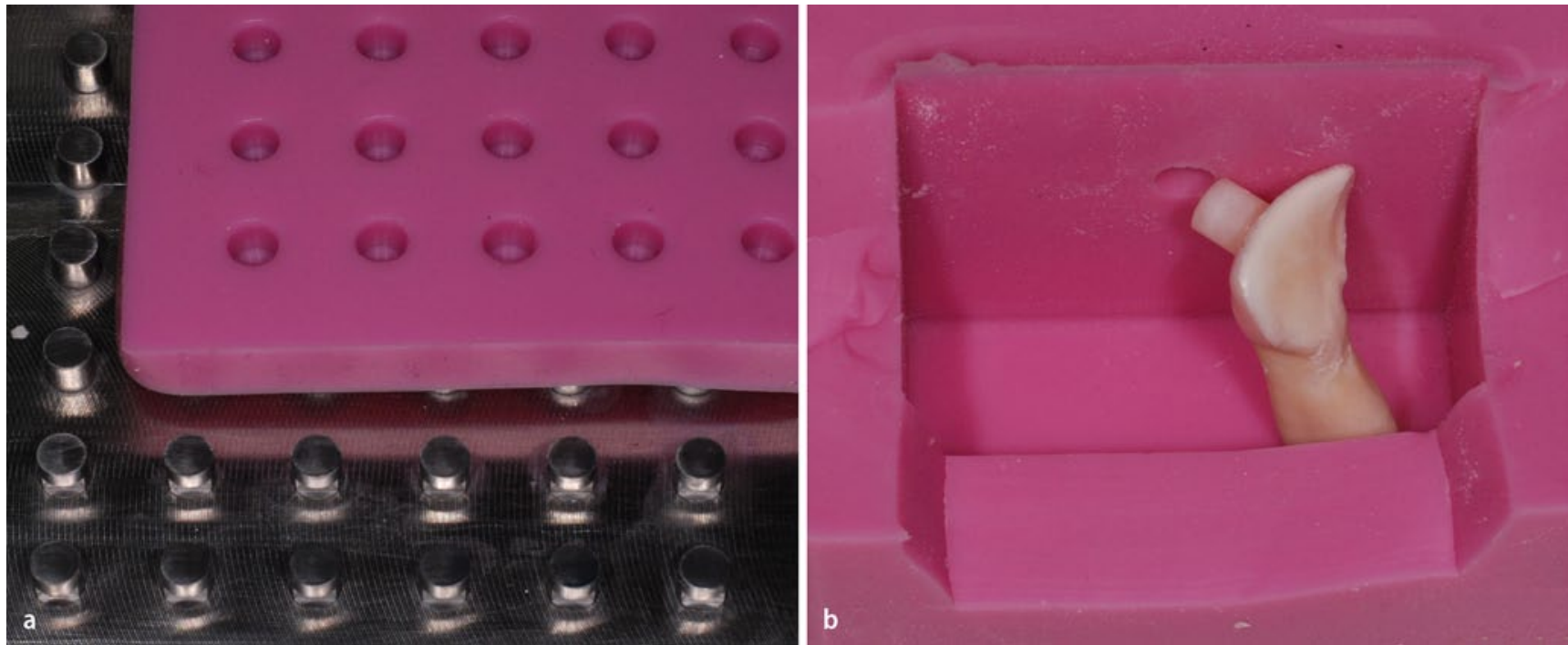

Figure 1. Specimen fabrication: the shear bodies were made by pouring a silicone mold of a CNC milled steel plate (a). A special singlebed form (b) allowed the shearing cylinder to be oriented parallel to the base surface of the test specimen

Abbildung 1. Probenherstellung: Die Abscherkörper wurden durch Ausgießen einer Silikonabformung einer CNC-gefrästen Stahlplatte erstellt (a). Eine spezielle Einbettform (b) ermöglichte die parallele Ausrichtung des Scherzylinders zur Grundfläche des Prüfkörpers

\section{Material and methods}

The shear strengths of seven self-etching primers were studied and compared with conventionally etched groups: Transbond $^{\mathrm{TM}} \mathrm{SEP}+\left(3 \mathrm{M}\right.$ Unitek, Monrovia, CA, USA), Xeno ${ }^{\circledR}$ III (Dentsply, Konstanz, Germany), Clearfil ${ }^{\mathrm{TM}} \mathrm{SE}$ (Kuraray, Frankfurt, Germany), Futurabond ${ }^{\circledR}$ NR (VOCO, Cuxhaven, Germany), One Coat (Coltène/Whaledent, Cuyahoga Falls, $\mathrm{OH}$, USA), M-Bond (Tokuyama, Burlingame, CA, USA) and OptiBond $^{\circledR}$ (Kerr GmbH, Bioggio, Switzerland). Each group consisted of 20 specimens, and the self-etching primers were administered observing manufacturer instructions. The selfetching primers were compared with two conventionallyetched groups using 35\% orthophosphoric acid (Unitek ${ }^{\mathrm{TM}}$ Etching Gel; 3M Unitek, Monrovia, CA, USA) and Transbond ${ }^{\mathrm{TM}}$ MIP.

Bovine incisors were used for the enamel substrate in compliance with ISO 11405/TS. The only teeth used were those whose macroscopic enamel surfaces were in perfect condition. The dental pulp was removed and the teeth stored temporarily prior to the shear tests in physiological saline solution.

We used shear bodies made of Grandio Flow (Grandio Flow; VOCO, Cuxhaven, Germany) in a cylindrical form with a base surface of $12.6 \mathrm{~mm}^{2}$ in all the specimens. For this purpose, a CNC milling machine (Picomax 60-M/HSC; Fehlmann AG, Seon, Switzerland) was used to mill a stainless-steel positive form base plate with 49 cylinders protruding from it. Using fastflowing silicone (Finosil; Fino GMBH, Schweinfurt, Germany) the cylinders were molded, creating a negative form. The hollow cylindrical forms in the silicone mold were filled with Grandio Flow to cast the shear bodies (Figure 1). Finally, the composite haftwerte auf ungeschliffenen Schmelzoberflächen nach Thermozyklierung. Ziel der Studie war weniger die erneute Überprüfung des bewährten Primers Transbond ${ }^{\mathrm{TM}} \mathrm{SEP}+$, sondern vielmehr die Eignung alternativer Primer zur kieferorthopädischen Anwendung.

\section{Material und Methodik}

Die Scherhaftkräfte von 7 selbstätzenden Primern wurden untersucht und mit konventionell geätzten Gruppen verglichen: Transbond ${ }^{\mathrm{TM}}$ SEP+ (3M Unitek, Monrovia, CA, USA), Xeno $^{\circledR}$ III (Dentsply, Konstanz, Deutschland), Clearfil ${ }^{\text {TM }}$ SE (Kuraray, Frankfurt, Deutschland), Futurabond ${ }^{\circledR}$ NR (VOCO, Cuxhaven, Deutschland), One Coat (Coltène/Whaledent, Cuyahoga Falls, Ohio, USA), M-Bond (Tokuyama, Burlingame, CA, USA) und OptiBond ${ }^{\circledR}$ (Kerr GmbH, Bioggio, Schweiz). Jede Gruppe bestand aus 20 Proben, die selbstätzenden Primer wurden gemäß Herstellerangaben appliziert. Die selbstätzenden Primer wurden mit 2 konventionell geätzten Gruppen unter Verwendung einer 35\%igen Orthophosphorsäure (Unitek ${ }^{\mathrm{TM}}$ Etching Gel; 3M Unitek, Monrovia, CA, USA) und Transbond ${ }^{\mathrm{TM}}$ MIP verglichen.

Als Schmelzsubstrat wurden gemäß ISO 11405/TS Rinderinzisivi verwendet. Es wurden ausschließlich Zähne mit makroskopisch einwandfreien Schmelzflächen verwendet. Die Pulpa der Zähne wurde entfernt und die Zähne bis zur Schertestung in physiologischer Kochsalzlösung zwischengelagert.

Für alle Proben wurden Abscherkörper aus Grandio Flow (Grandio Flow; VOCO, Cuxhaven, Deutschland) mit zylindrischer Form und einer Grundfläche von $12,6 \mathrm{~mm}^{2}$ verwendet. Dazu wurde über eine CNC-Fräse (Picomax 60-M/HSC; Fehlmann 


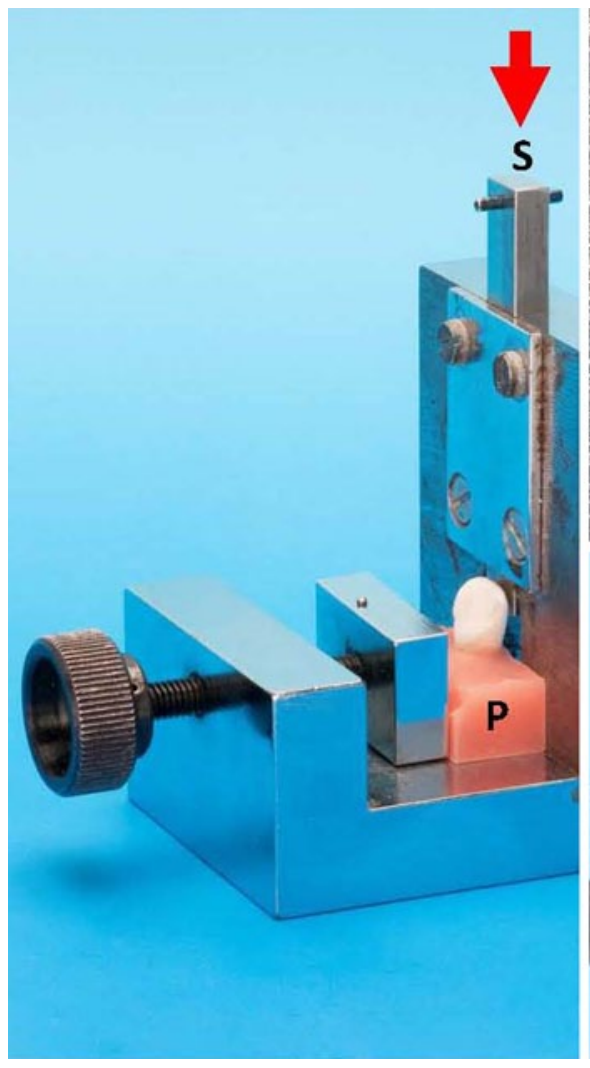

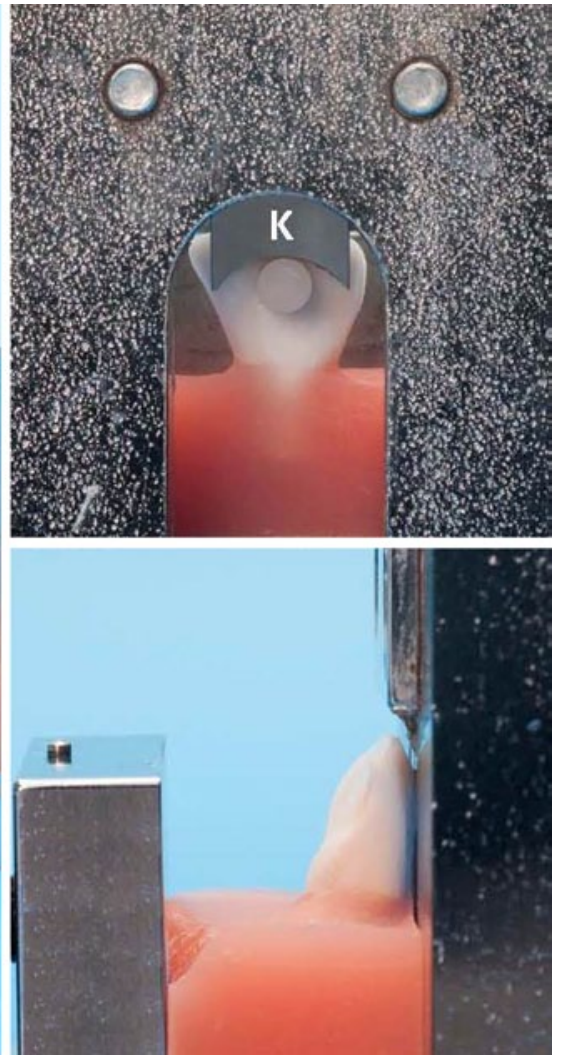

Figure 2. Shear assembly: the overview shows the shear plunger $(S)$, specimen $(P)$ and direction of pressure of the universal testing machine (arrow). The detailed images illustrate the form of the shear blade $(K)$, the minimum clearance and its parallelism to the enamel surface

Abbildung 2. Schervorrichtung: Die Übersicht zeigt den Scherstempel (S), die Probe (P) und die Druckrichtung der Universalprüfmaschine (Pfeil). Auf den Detailaufnahmen sind die Gestaltung der Scherklinge (K) sowie deren minimaler Abstand und Parallelität zur Schmelzoberfläche ersichtlich cylinders were light-cured for $40 \mathrm{~s}$ at $800 \mathrm{~mW} / \mathrm{cm}^{2}$ (bluephase $^{\circledR}$ C8; Ivoclar Vivadent, Schaan, Liechtenstein).

After bonding the composite cylinders onto the prepared enamel specimens using Transbond ${ }^{\mathrm{TM}}$ XT (3M Unitek, Monrovia, CA, USA) composite, and another curing period of $40 \mathrm{~s}$ (bluephase ${ }^{\circledR} \mathrm{C} 8$; Ivoclar Vivadent, Liechtenstein), we placed all the self-etching primer samples and one of the two control groups in a contrast bath set at 1000 thermo-cycles between 5 and $55^{\circ} \mathrm{C}$ for a period of $50 \mathrm{~h}$ [Circulator C-85 (Techne, Brouwer, Luzern, Switzerland) and Julabo UC and 5B (Julabo Labortechnik, Seelbach, Germany)]. The second control group was not thermo-cycled.

We ensured that the enamel adhesive bond was parallel to the shear direction by using a custom-made single-bed form (Figure 1). Polymethacrylate was poured around the roots of the teeth (Technovit; Heraeus Kulzer, Wehrheim, Germany) and the resulting specimens were sheared off using a universal testing machine (Instron 4444; Instron Corp., Wilmington, DE, USA). The distance of the shear blade from the enamel surface was set at $0.5 \mathrm{~mm}$ (Figure 2). These data were recorded automatically at a shear speed of $0.1 \mathrm{~mm} / \mathrm{s}$ on a PC.

The adhesive remnant index (ARI) developed by Artun [2] was documented at a 3.5-fold magnification in all specimens.

Statistical evaluation of the adhesive strengths (Figure 2) was carried out using GraphPad Instat 3 (GraphPad Software Inc., San Diego, CA, USA). Normal distribution was calculated according to Kolmogorov-Smirnov. The descriptive statistics were
AG, Seon, Schweiz) aus Edelstahl eine Positivform-Grundplatte mit 49 daraus hervorragenden Zylindern gefräst. Mittels eines dünnfließenden Silikons (Finosil; Fino GMBH, Schweinfurt, Deutschland) wurden die Zylinder abgeformt und eine Negativform erstellt. Die Zylinderhohlformen in der Silikonabformung wurden zur Herstellung der Abscherkörper mit Grandio Flow ausgegossen (Abbildung 1). Abschließend erfolgte eine Lichthärtung der Kompositzylinder über $40 \mathrm{~s}$ bei $800 \mathrm{~mW} / \mathrm{cm}^{2}$ (bluepha$\mathrm{se}^{\circledR} \mathrm{C}$; Ivoclar Vivadent, Schaan, Liechtenstein).

Nach Bonding der Kompositzylinder auf die vorbereiteten Schmelzproben mittels des Komposits Transbond ${ }^{\mathrm{TM}}$ XT (3M Unitek, Monrovia, CA, USA) und erneuter Härtung für $40 \mathrm{~s}$ (bluephase $^{\circledR}$ C8; Ivoclar Vivadent, Liechtenstein) wurden sämtliche Proben der selbstätzenden Primer und eine der beiden Kontrollgruppen in einem Wechselbad einer 1000-fachen Thermozyklierung von $5-55^{\circ} \mathrm{C}$ über eine Laufzeit von $50 \mathrm{~h}$ ausgesetzt (Circulator C-85, Techne, Brouwer, Luzern, Schweiz; Julabo UC und 5B, Julabo Labortechnik, Seelbach, Deutschland). Die zweite Kontrollgruppe wurde nicht thermozykliert.

Die Parallelität der Schmelzadhäsivverbindung mit der Scherrichtung wurde durch die Verwendung einer eigens gefertigten Einbettform gewährleistet (Abbildung 1). Die Wurzeln der Zähne wurden mit einem Polymethacrylat umgossen (Technovit; Heraeus Kulzer, Wehrheim, Deutschland) und die so hergestellten Proben mit einer Universalprüfmaschine (Instron 4444; Instron Corp., Wilmington, DE, USA) abgeschert. Der Abstand der Scherklinge zur Schmelzoberfläche wurde auf 

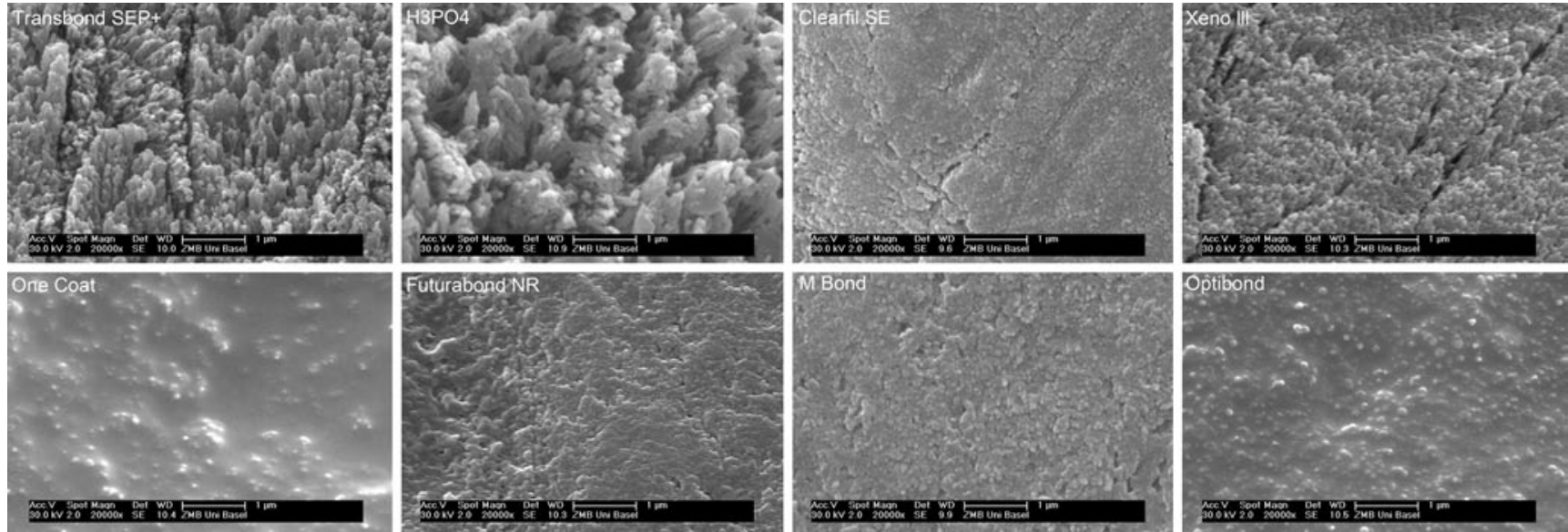

Figure 3. Enamel surfaces after etching with various primers as well as $\mathrm{H}_{3} \mathrm{PO}_{4}$. Obvious differences in quality are visible in the depth of the etching relief. The sequence corresponds to the shear strengths achieved

Abbildung 3. Schmelzoberflächen nach Ätzung mit unterschiedlichen Primern, sowie $\mathrm{H}_{3} \mathrm{PO}_{4}$. Es sind deutliche qualitative Differenzen in der Ausprägung des Ätzreliefs ersichtlich. Die Reihenfolge entspricht den erreichten Scherkräften

performed calculating the mean, median, standard deviation and the standard error of the mean. The Kruskal-Wallis and Dunn's post-hoc test with a significance level set at $\mathrm{p} \leq 0.05$ were used for the comparative statistics.

In addition, the $\mathrm{pH}$ value of all the self-etching primers was measured using test strips featuring 0.5-stage $\mathrm{pH}$ segments (Acilit pH 0-6; Merck KGaG, Darmstadt, Germany). Furthermore, we examined the etching patterns of three additional specimens of each etchant under a scanning electron microscope in a vacuum at 20,000-fold magnification (ESEM, Philips 30; Royal Philips Electronics, The Netherlands), capturing typical sections (Figure 3).

\section{Results}

Our results are shown in Table 1, Table 2, Figure 3 and Figure 4.

The difference in the adhesion values of the control group with thermo-cycling (x $32.3 \mathrm{MPa}$ ) and without thermo-cycling $(\overline{\mathrm{x}}=35.5 \mathrm{MPa})$ was very small and statistically not significant (Table 1, Figure 2). Transbond ${ }^{\mathrm{TM}} \mathrm{SEP}+$ revealed nearly identical values $(\overline{\mathrm{X}}=34.2 \mathrm{MPa})$. Even the difference compared to Clear$\mathrm{fil}^{\mathrm{TM}} \mathrm{SE}(\mathrm{x}=26.8 \mathrm{MPa})$ was statistically insignificant. The remaining self-etching primers demonstrated significantly lower adhesive values $\left(\mathrm{Xeno}^{\circledR}\right.$ III: $\overline{\mathrm{X}}=15.2 \mathrm{MPa}$, One Coat: $\overline{\mathrm{X}}=11.5$ $\mathrm{MPa}$, Futurabond ${ }^{\circledR}$ NR: $\overline{\mathrm{x}}=9.0 \mathrm{MPa}, \mathrm{M}$-Bond: $\overline{\mathrm{x}}=8.6 \mathrm{MPa}$, OptiBond $^{\circledR}: \overline{\mathrm{X}}=0.0 \mathrm{MPa}$ ). All groups aside from the control group and Transbond ${ }^{\text {TM }}$ SEP+, presented spontaneous losses from the shear bodies during thermo-cycling. The frequency of adhesion losses was inversely proportional to the adhesion values. In the OptiBond ${ }^{\circledR}$ group, all the specimens suffered losses from shear bodies during thermo-cycling, thus that group could no longer undergo evaluation. No etching relief was visible when the specimens with shear body losses were inspected.
0,5 mm festgelegt (Abbildung 2). Die Datenaufzeichnung erfolgte automatisiert bei einer Schergeschwindigkeit von 0,1 $\mathrm{mm} / \mathrm{s}$ über einen PC.

Für sämtliche Proben wurde unter 3,5-facher Vergrößerung der Adhäsiv-Rest-Index (ARI) nach Artun [2] festgehalten.

Die statistische Auswertung der Haftkräfte (Tabelle 1) und der ARI-Werte (Tabelle 2) erfolgte mithilfe der Software GraphPad Instat 3 (GraphPad Software Inc., San Diego, CA, USA). Die Normalverteilung wurde nach Kolmogorov-Smirnov berechnet. Die deskriptive Statistik erfolgte unter Berechnung von Mittelwert, Median, Standardabweichung und Standardmittelwertfehler. Die vergleichende Statistik wurde mit dem KruskalWallis-Test und dem Post-hoc-Test nach Dunn durchgeführt, wobei das Signifikanzniveau auf $\mathrm{p} \leq 0,05$ festgelegt wurde.

Zusätzlich wurden von allen selbstätzenden Primern $\mathrm{pH}$ Messungen mittels Teststreifen mit 0,5-stufiger $\mathrm{pH}$-Einteilung (Acilit pH 0-6; Merck KGaG, Darmstadt, Deutschland) durchgeführt. Außerdem wurden die Ätzmuster von jeweils 3 zusätzlichen Proben im Rasterelektronenmikroskop unter Vakuum und bei 20.000-facher Vergrößerung dargestellt (ESEM, Philips 30; Royal Philips Electronics, Niederlande) und typische Bereiche festgehalten (Abbildung 3).

\section{Ergebnisse}

Die Resultate sind übersichtlich in Tabelle 1 und Tabelle 2 sowie in Abbildung 3 und Abbildung 4 dargestellt.

Der Unterschied der Haftwerte der Kontrollgruppen ohne Thermozyklierung $(\overline{\mathrm{x}}=35,5 \mathrm{MPa})$ und mit Thermozyklierung $(\overline{\mathrm{X}}=32,3 \mathrm{MPa})$ war sehr klein und statistisch nicht signifikant (Tabelle 1, Abbildung 2). Transbond ${ }^{\mathrm{TM}} \mathrm{SEP}+$ wies fast identische Werte auf $(\overline{\mathrm{x}}=34,2 \mathrm{MPa})$. Auch der Unterschied zu Clearfil ${ }^{\mathrm{TM}} \mathrm{SE}$ $(\overline{\mathrm{x}}=26,8 \mathrm{MPa})$ war statistisch nicht signifikant. Die restlichen selbstätzenden Primer zeigten signifikant verringerte Haftwerte 
Table 1. Shear strengths of the seven self-etching (SE) primers in comparison with the control group Transbond ${ }^{\mathrm{TM}} \mathrm{XT}$. Mean values, standard deviation (SD), standard error of the mean (SEM), median, normal distribution according to Kolmogorov-Smirnov, ranking, significance and spontaneous detachments after thermo-cycling

Tabelle 1. Scherkräfte der 7 SE-Primer im Vergleich zur Kontrollgruppe Transbond ${ }^{\mathrm{TM}}$ XT. Mittelwerte, Standardabweichung (SD), Mittelwertfehler (SEM), Median, Normalverteilung nach Kolmogorov-Smirnov, Rangfolge, Signifikanz und spontane Haftverluste nach Thermozyklierung

\begin{tabular}{|c|c|c|c|c|c|c|c|c|c|}
\hline Primer & $\begin{array}{l}\text { Group } \\
\mathrm{N \#}\end{array}$ & $\begin{array}{l}\text { Mean } \\
(\mathrm{MPa})\end{array}$ & SD & SEM & $\begin{array}{l}\text { Median } \\
\text { (MPa) }\end{array}$ & $\begin{array}{l}\text { Kolmogorov- } \\
\text { Smirnov, } \\
\text { p value }\end{array}$ & $\begin{array}{l}\text { Ranking } \\
\text { (Kruskal- } \\
\text { Wallis) }\end{array}$ & $\begin{array}{l}\text { Significance } \\
\text { (Dunn, } \\
\mathrm{p} \leq 0.05 \text { ) }\end{array}$ & $\begin{array}{l}\text { Spontaneous } \\
\text { detachments }\end{array}$ \\
\hline $\begin{array}{l}\text { Transbond }{ }^{\mathrm{TM}} \text { MIP } \\
\text { No thermo cycles }\end{array}$ & 1 & 35.3 & 13.4 & 3.0 & 36.2 & $>0.1$ & 1 & $4,6,7,8,9$ & 0 \\
\hline $\begin{array}{l}\text { Transbond }{ }^{\mathrm{TM}} \text { MIP } \\
\text { With thermo cycles }\end{array}$ & 2 & 32.3 & 7.2 & 1.6 & 32.6 & $>0.1$ & 1 & $4,6,7,8,9$ & 0 \\
\hline Transbond $^{\mathrm{TM}}$ SEP plus & 3 & 34.2 & 7.3 & 1.6 & 34.7 & $>0.1$ & 1 & $4,6,7,8,9$ & 0 \\
\hline Xeno $^{\circledast}$ III & 4 & 15.2 & 3.2 & 0.8 & 15.2 & $>0.1$ & 3 & $1,2,3$ & 5 \\
\hline Clearfil $^{\mathrm{TM}} \mathrm{SE}$ & 5 & 26.8 & 7.7 & 1.8 & 27 & 0.1 & 2 & $6,7,8,9$ & 1 \\
\hline Futurabond ${ }^{\circledR} \mathrm{NR}$ & 6 & 9.0 & 4.2 & 1.1 & 7.9 & $>0.1$ & 4 & $1,2,3,5$ & 7 \\
\hline One Coat & 7 & 11.5 & 9.8 & 2.5 & 11.1 & 0.0001 & 4 & $1,2,3,5$ & 5 \\
\hline M-Bond & 8 & 8.6 & 3.7 & 1.0 & 6.6 & $>0.1$ & 4 & $1,2,3,5$ & 7 \\
\hline OptiBond ${ }^{\circledR}$ & 9 & 0.0 & - & - & - & - & 5 & $1-8$ & 20 \\
\hline
\end{tabular}

All groups except the One Coat group demonstrated normal distribution in the statistical evaluation. OptiBond ${ }^{\circledR}$ was not analyzed.

The ARI correlated closely with the adhesion values measured, while the specimens with high adhesion values revealed a high ARI (Table 2). Statistical analysis of the ARI yielded a significant difference among the three Transbond ${ }^{\mathrm{TM}}$ products and remaining self-etching primers, which did not significantly differ from one another. Optibond ${ }^{\circledR}$ was not included in the analysis. Enamel tear-outs were only observed in the Transbond ${ }^{\mathrm{TM}}$ groups; they also showed agreement with the adhesion values obtained.

The $\mathrm{pH}$ measurements differed considerably: Transbond ${ }^{\mathrm{TM}}$ $\mathrm{SEP}+$ and $\mathrm{Xeno}^{\circledR} \mathrm{III}$ revealed the lowest $\mathrm{pH}$ values $(\mathrm{pH}=1)$, followed by Futurabond ${ }^{\circledR} \mathrm{NR}$ and $\mathrm{M}$-Bond $(\mathrm{pH}=1.5)$ and Clearfil $^{\text {TM }} \mathrm{SE}$, One Coat and OptiBond ${ }^{\circledR}(\mathrm{pH}=2)$.

Conventional etching and Transbond ${ }^{\mathrm{TM}} \mathrm{SEP}+$ demonstrated the most obvious etching patterns. Xeno ${ }^{\circledR}$ III also showed clear etching patterns. Surface roughness from the remaining primers was only moderate; OptiBond ${ }^{\circledR}$ and One Coat revealed hardly any etching pattern (Figure 4).

\section{Discussion}

The outer layer of enamel displayed some differences compared to the deeper enamel layers. Morphologically this consists of a non-prismatic layer $[40,50]$ which appears poorly suited for adhesive procedures $[27,32]$ and especially for the use of self-etching primers $[10,27,37]$. In addition, the outer enamel layer contains a modified ratio of fluorapatite and hydroxyapatite, whereby the more acid-resistant fluorapatite occupies a higher percentage [45]. The etching of the surface
(Xeno $^{\circledR}$ III: $\overline{\mathrm{X}}=15,2 \mathrm{MPa}$, One Coat: $\overline{\mathrm{X}}=11,5 \mathrm{MPa}$, Futurabond ${ }^{\circledR}$ NR: $\overline{\mathrm{X}}=9,0 \mathrm{MPa}, \mathrm{M}$-Bond: $\overline{\mathrm{X}}=8,6 \mathrm{MPa}$, OptiBond ${ }^{\circledR}: \overline{\mathrm{X}}=0,0$ $\mathrm{MPa})$. Alle Gruppen außer der Kontrollgruppe und Transbond ${ }^{\text {TM }}$ SEP + wiesen spontane Verluste von Scherkörpern während der Thermozyklierung auf. Die Häufigkeit der Haftverluste stand in umgekehrt proportionalem Verhältnis zu den Haftwerten. In der OptiBond ${ }^{\circledR}$-Gruppe erlitten sämtliche Proben Verluste von Scherkörpern unter Thermozyklierung, weshalb die Gruppe nicht weiter ausgewertet werden konnte. In der visuellen Überprüfung der Proben mit Scherkörperverlusten war kein Ätzrelief ersichtlich.

Die statistische Auswertung der Daten ergab für alle Gruppen außer One Coat eine Normalverteilung. OptiBond ${ }^{\circledR}$ wurde nicht ausgewertet.

Der ARI wies eine deutliche Abhängigkeit von den gemessenen Haftwerten auf, wobei sich für Proben mit hohen Haftwerten ein hoher ARI ergab (Tabelle 2). Die statistische Auswertung des ARI ergab einen signifikanten Unterschied zwischen den 3 Transbond ${ }^{\text {TM }}$-Produkten und den restlichen selbstätzenden Primern, die sich ihrerseits nicht signifikant voneinander unterschieden. OptiBond ${ }^{\circledR}$ wurde nicht in die Auswertung einbezogen. Schmelzausrisse wurden nur in den Transbond ${ }^{\mathrm{TM}}$-Gruppen beobachtet und wiesen ebenfalls eine Übereinstimmung mit den erzielten Haftwerten auf.

Die $\mathrm{pH}-\mathrm{Messungen}$ ergaben deutliche Unterschiede: Transbond $^{\text {TM }} \mathrm{SEP}+$ und $\mathrm{Xeno}^{\circledR}$ III wiesen die niedrigsten $\mathrm{pH}$-Werte auf $(\mathrm{pH}=1)$. Es folgten Futurabond ${ }^{\circledR} \mathrm{NR}$ und $\mathrm{M}$-Bond $(\mathrm{pH}=1,5)$ sowie Clearfil ${ }^{\mathrm{TM}} \mathrm{SE}$, One Coat und OptiBond ${ }^{\circledR}(\mathrm{pH}=2)$.

Die konventionelle Ätzung sowie Transbond ${ }^{\mathrm{TM}} \mathrm{SEP}+$ wiesen die ausgeprägtesten Ätzstrukturen auf. Auch $\mathrm{Xeno}^{\circledR}$ III zeigte deutliche Ätzmuster. Die Oberflächenaufrauung der restlichen 
Table 2. Adhesive remnant index (ARI): mean values, standard deviation, ranking and significance. The ARI values correlate closely with the shear strengths measured

Tabelle 2. Adhäsiv-Rest-Index (ARI): Mittelwerte, Standardabweichung, Rangfolge und Signifikanz. Die ARI-Werte standen in engem Zusammenhang mit den gemessenen Scherkräften

\begin{tabular}{llllll}
\hline & $\begin{array}{l}\text { Group } \\
\text { N\# }\end{array}$ & Mean & Enamel fracture & $\begin{array}{l}\text { Rank } \\
\text { (Kruskal-Wallis) }\end{array}$ & $\begin{array}{l}\text { Significance } \\
\text { (Dunn, } \mathbf{p} \leq 0.05)\end{array}$ \\
\hline Transbond $^{\text {TM }}$ & 1 & 2.7 & 9 & 1 & $4-9$ \\
Transbond $^{\text {TM }}$ TC & 2 & 2.2 & 5 & 1 & $4-9$ \\
Transbond $^{\text {TM }}$ SEP & 3 & 2.1 & 3 & 1 & $4-9$ \\
Xeno $^{\oplus}$ III & 4 & 0.4 & 0 & 2 & $1-3,9$ \\
Clearfil' $^{\text {TM }}$ SE & 5 & 0.8 & 0 & 2 & $1-3,9$ \\
Futurabond $^{\oplus}$ NR & 6 & 0.3 & 0 & 2 & $1-3,9$ \\
One Coat & 7 & 0.6 & 0 & 2 & $1-3,9$ \\
M-Bond & 8 & 0.5 & 0 & 2 & $1-3,9$ \\
OptiBond $^{\circledast}$ & 9 & 0.0 & 0 & 3 & $1-8$
\end{tabular}

enamel with weak acids may thus be reduced, as confirmed in several studies [23,37] and rejected in another [37]. This study has not enabled us to provide a definitive answer to this question either. On the one hand, Transbond ${ }^{\mathrm{TM}} \mathrm{SEP}+(\mathrm{pH}=1)$ revealed better adhesion values than primers with higher $\mathrm{pH}$ values such as Futurabond ${ }^{\circledast}(\mathrm{pH}=1.5)$, One Coat $(\mathrm{pH}=2)$, $\mathrm{M}$ Bond $(\mathrm{pH}=1.5)$ and OptiBond ${ }^{\circledR}(\mathrm{pH}=2)$, but Clearfil ${ }^{\mathrm{TM}} \mathrm{SE}$ $(\mathrm{pH}=2)$ obtained very high adhesion values-clearly ahead of $\mathrm{Xeno}^{\circledR}$ III $(\mathrm{pH}=1)$. The good adhesion strength of Clearfil ${ }^{\mathrm{TM}}$ with a relatively high $\mathrm{pH}$ value was confirmed in an earlier study [34] and is probably related to the adhesive components used, 10-MDP [48]. From studies on the correlation between adhesion values and the depth of etching relief, we learned that the infiltration depth of the primer is more important than just the depth of the etching pattern $[8,22,44]$.

We observed no clear correlation between adhesion values and the acids used. Most primers contain phosphoric acid ester (Transbond $^{\mathrm{TM}}$ SEP $+\mathrm{XENO}^{\circledR}$ III, Clearfil ${ }^{\mathrm{TM}}$ SE, Futurabond ${ }^{\circledR}$ NR, M-Bond). Only OptiBond ${ }^{\circledR}$ (methylic acid) and One Coat (acrylamide sulfonic acid) used other groups of acid. While we noted deep but not significantly different adhesion values in association with M-Bond and One Coat, OptiBond ${ }^{\circledR}$ could not be analyzed due to complete adhesion failure during thermo-cycling. The barely perceptible etching patterns caused by One Coat and OptiBond ${ }^{\circledR}$ (Figure 4) were striking. These substances are apparently either not, or only partially, suitable for use on surface enamel.

The specimens in the present study were exposed to 1000 -fold thermo-cycling between 5 and $55^{\circ} \mathrm{C}$, which according to earlier studies can lead to reduced adhesion values $[16,18]$. One can assume that this enables a better approximation of the intraoral situation [4]. The control group without thermo-cycling acted as a control for the load procedure. The considerable drop in adhesion strength after thermo-cycling could be attributed to overload caused by excessive thermal fluctuations. However, in
Primer war nur mäßig ausgeprägt, bei OptiBond ${ }^{\circledR}$ und One Coat zeigte sich kaum eine Ätzstruktur (Abbildung 4).

\section{Diskussion}

Die äußere Schmelzschicht weist einige Unterschiede zu tiefer liegenden Schmelzschichten auf. Morphologisch besteht sie aus einer aprismatischen Schicht [40, 50], die für Haftverfahren [27, 32] und insbesondere die Verwendung von selbstätzenden Primern ungünstig erscheint $[10,27,37]$. Zudem enthält die äußere Schmelzschicht ein verändertes Verhältnis von Fluorapatit und Hydroxylapatit, wobei säureresistenter Fluorapatit zu einem erhöhten Prozentsatz vorkommt [45]. Die Ätzung von oberflächlichem Schmelz mit schwachen Säuren könnte deshalb vermindert ausfallen, was in einigen Studien bestätigt werden konnte $[23,37]$ und in einer anderen verworfen wurde [37]. Auch die vorliegende Arbeit kann keine abschließende Antwort liefern. Transbond ${ }^{T M} \mathrm{SEP}+(\mathrm{pH}=1)$ wies zwar bessere Haftwerte als Primer mit höheren $\mathrm{pH}-$ Werten wie Futurabond ${ }^{\circledR}(\mathrm{pH}=1,5)$, One Coat $(\mathrm{pH}=2), \mathrm{M}$-Bond $(\mathrm{pH}=1,5)$ und OptiBond ${ }^{\circledast}(\mathrm{pH}=2)$ auf, allerdings erreichte Clearfil ${ }^{\mathrm{TM}} \mathrm{SE}(\mathrm{pH}=2)$ sehr hohe Haftwerte, die deutlich über denen von Xeno III $(\mathrm{pH}=1)$ lagen. Die gute Haftkraft von Clearfil ${ }^{\mathrm{TM}}$ bei relativ hohem $\mathrm{pH}$-Wert fand sich in einer früheren Studie bestätigt [34] und steht wahrscheinlich im Zusammenhang mit der verwendeten Adhäsivkomponente 10-MDP [48]. In Studien zur Korrelation der Haftwerte mit der Tiefe des Ätzreliefs konnte gezeigt werden, dass die Infiltrationstiefe des Primers wichtiger ist als die alleinige Tiefe des Ätzmusters [8, 22, 44].

Des Weiteren konnte keine klare Korrelation zwischen Haftwerten und verwendeter Säure gefunden werden. Die meisten Primer enthielten Phosphorsäureester (Transbond ${ }^{\mathrm{TM}} \mathrm{SEP}+$, Xeno $^{\circledast}$ III, Clearfil ${ }^{T M}$ SE, Futurabond ${ }^{\circledR}$ NR, M-Bond). Nur OptiBond $^{\circledast}$ (Methylsäure) und One Coat (Acrylamidsulfonsäure) verwendeten andere Säuregruppen. Während für M-Bond und 


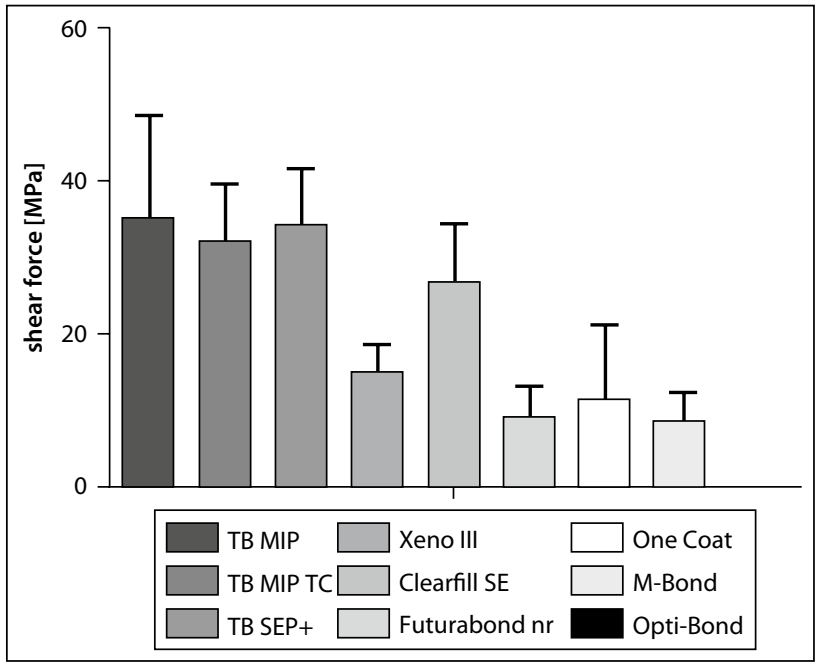

Figure 4. Shear strengths shown as mean values and standard deviations. As all specimens of the primer OptiBond ${ }^{\circledR}$ displayed spontaneous adhesion losses after thermo-cycling, shear strengths could not be measured

Abbildung 3. Scherkräfte, dargestellt als Mittelwerte und Standardabweichungen. Bei allen Proben des Primers OptiBond ${ }^{\circledR}$ fanden sich spontane Haftverluste nach der Thermozyklierung, weshalb keine Scherkräfte gemessen werden konnten

comparison to the control groups with and without thermo-cycling, we observed no significant adhesion loss. We thus assume that the thermo-cycling range we employed was reasonable. Unlike earlier studies without thermo-cycling, several self-etching primers showed unreliable adhesion values $[1,8,10]$ - the number of spontaneous adhesion losses caused by thermo-cycling was particularly astonishing. Besides the Transbond ${ }^{\mathrm{TM}}$ products, only Clearfil ${ }^{\mathrm{TM}} \mathrm{SE}$ revealed acceptable stress behavior with a single spontaneous fracture, while all the OptiBond ${ }^{\circledR}$ specimens displayed complete adhesion loss during thermocycling. The clinical applicability of the products with high spontaneous adhesion losses is questionable. Adhesion loss in the oral cavity is caused by thermal fluctuations and repetitive mechanical loads, fluid absorption, and biodegradation [10, 24, $28,31]$. Hence, adhesion losses of as high as $30 \%$ have been measured over a period of 6 months [19].

The ARI values observed in this study agree well with the adhesion values we obtained. While the Transbond ${ }^{\text {TM }}$ groups' values fell between 2.1 and 2.7, indicating cohesive failure of the adhesive bond, the self-etching primers with deep adhesion strengths showed ARI values $\leq 0.8$, demonstrating a weak point in the enamel-adhesive bond (Table 2). Our Transbond ${ }^{\mathrm{TM}}$ groups' ARI values and adhesion values with over $30 \mathrm{MPa}$ are thus clearly higher than findings in the literature, which reveal adhesive failure in the bracket-composite interface [10, 43]. This may be related to the shear bodies used. When brackets are used, the adhesive break usually occurs between the bracket base and composite $[3,17]$. This is unfavorable when testing enamel adhesion using self-etching primers, as what is being tested instead is the adhesion of the resin to the bracket base. We circumvented
One Coat tiefe, aber nicht signifikant unterschiedliche Haftwerte vorlagen, konnte OptiBond ${ }^{\circledR}$ aufgrund der kompletten Haftverluste während der Thermozyklierung nicht weiter ausgewertet werden. Auffällig waren die kaum ausgeprägten Ätzmuster bei One Coat und OptiBond ${ }^{\circledR}$ (Abbildung 4). Die Substanzen scheinen für die Anwendung auf oberflächlichem Schmelz nicht oder nur bedingt geeignet.

Die Proben wurden in der vorliegenden Untersuchung einer 1000 -fachen Thermozyklierung von $5-55^{\circ} \mathrm{C}$ unterzogen, was früheren Studien gemäß zu einer Minderung der Haftwerte führen kann $[16,18]$. Es ist davon auszugehen, dass die intraorale Situation dadurch besser repräsentiert wird [4]. Die Kontrollgruppe ohne Thermozyklierung diente der Überprüfung des Belastungsverfahrens. Ein starker Abfall der Haftkraft nach Thermozyklierung könnte auf eine Überlastung durch exzessive thermische Wechselspannungen zurückzuführen sein. Im Vergleich der Kontrollgruppen mit und ohne Thermozyklierung konnte allerdings kein signifikanter Haftverlust festgestellt werden. Es ist deshalb davon auszugehen, dass die angewandte Thermozyklierung in einem sinnvollen Bereich liegt. Demgegenüber wiesen einige selbstätzende Primer im Vergleich zu früheren Studien ohne Thermozyklierung ungünstige Haftwerte auf $[1,8,10]$. Erstaunlich war insbesondere die Anzahl spontaner Haftverluste durch die Thermozyklierung. Neben den Transbond ${ }^{\mathrm{TM}}$-Produkten zeigte nur Clearfil ${ }^{\mathrm{TM}}$ SE mit einer einzelnen spontanen Fraktur ein akzeptables Stressverhalten, während bei OptiBond ${ }^{\circledR}$ sämtliche Proben während den Thermozyklen einen kompletten Haftverlust aufwiesen. Die klinische Anwendbarkeit der Produkte mit hohen spontanen Haftverlusten ist fraglich. In der Mundhöhle führen neben thermischen Schwankungen auch repetitive mechanische Belastungen, Flüssigkeitsaufnahme und Biodegradation zu einem Haftverlust $[10,24,28,31]$. So wurden Haftverluste bis $30 \%$ über 6 Monate gemessen [19].

Die in der Studie gefundenen ARI-Werte standen in guter Übereinstimmung mit den gefundenen Haftwerten. Während die Transbond ${ }^{\mathrm{TM}}$-Gruppen Werte zwischen 2,1-2,7 aufwiesen, die auf ein kohäsives Versagen des Haftverbunds hindeuten, zeigten selbstätzende Primer mit tiefen Haftkräften ARI-Werte $\leq 0,8$, was auf eine Schwachstelle im Schmelz-Adhäsiv-Verbund hindeutet (Tabelle 2). Die ARI-Werte der Transbond ${ }^{\mathrm{TM}}$-Gruppe und die Haftwerte von über $30 \mathrm{MPa}$ lagen damit deutlich über den in der Literatur gefundenen Angaben, die auf ein adhäsives Versagen des Bracket-Komposit-Übergangs hindeuten [10, 43]. Dies könnte mit den verwendeten Scherkörpern zusammenhängen. Bei Verwendung von Brackets tritt meist ein adhäsiver Bruch zwischen Bracketbasis und Komposit auf [3, 17]. Für die Prüfung der Schmelzadhäsion mittels selbstätzender Primer ist dies ungünstig, da vielmehr die Adhäsion des verwendeten Kunststoffs zur Bracketbasis getestet wird. Die Problematik konnte mit den Kompositscherkörpern umgangen werden. Die z. T. sehr hohen Haftwerte mit großem Anteil an Schmelzausrissen dürften neben dem geringen Abstand der Scherklinge zur Schmelzoberfläche mit daraus folgender hoher Scher- und tiefer Schälkomponente auf der Unterbindung eines Frakturver- 
this problem by using composite shear bodies. Our occasionally very high adhesion values with a large percentage of enamel tear-outs are doubtless due to having prevented a fracture line between the bracket base and composite, in addition to the small distance between the shear blade and enamel surface and thus high shearing and deeper peeling components. Enamel tear-outs were only observed in the widely-used Transbond ${ }^{\mathrm{TM}}$ products, and only in conjunction with forces having more than twice the cohesion strength of enamel (14 MPa) [39]. The enamel tear-out problem is not known in the clinical context to this extent. In comparison to the present study, the adhesive bond in clinical use is not attained solely by shearing effect. Moreover, the adhesive bond is weakened before debonding by the aforementioned stress effects. Nevertheless, special care must be taken when debonding, especially when dealing with many selfligating brackets with a highly rigid base, or ceramic brackets that cannot be deformed and peeled off in response to wing compression.

\section{Conclusions}

Only Transbond ${ }^{\mathrm{TM}} \mathrm{SEP}+$ and Clearfil ${ }^{\mathrm{TM}} \mathrm{SE}$ achieved adhesive values corresponding to those of conventional adhesive techniques. The remaining self-etching primers failed to convince due to the high rate of complete adhesive failures during thermo-cycling.

\section{Acknowledgments}

We would like to thank the companies who provided us with the materials needed for the study free of charge.

\section{Conflict of interest}

The corresponding author states that there are no conflicts of interest. laufs zwischen Bracketbasis und Komposit beruhen. Schmelzausrisse wurden nur bei den klinisch weitverbreiteten Transbond ${ }^{\mathrm{TM}}$-Produkten und erst bei einer mehr als doppelt so hohen Kohäsionskraft des Schmelzes von $14 \mathrm{MPa}$ [39] beobachtet. Die Problematik der Schmelzausrisse ist in der klinischen Anwendung nicht in diesem Maße bekannt. Im Vergleich zur vorliegenden Untersuchung wird in der klinischen Verwendung der Haftverbund nicht ausschließlich durch eine reine Scherwirkung erreicht. Zudem wird der Haftverbund vor Debonding über bereits beschriebene Stresseinwirkungen reduziert. Dennoch sollte bei Debonding gerade bei vielen selbstligierenden Brackets mit einer sehr starren Basis bzw. bei Keramikbrackets, die sich nicht durch Kompression der Flügel verformen und abschälen lassen, auf ein vorsichtiges Vorgehen geachtet werden.

\section{Schlussfolgerung}

Nur Transbond ${ }^{\mathrm{TM}}$ SEP+ und Clearfil ${ }^{\mathrm{TM}}$ SE erreichten Haftwerte, die denen der konventionellen Klebetechniken entsprachen. Die restlichen selbstätzenden Primer konnten auch aufgrund der hohen vollständigen Haftverlustraten während der Thermozyklierung nicht überzeugen.

\section{Danksagung}

Wir möchten uns bei den Firmen bedanken, die uns die Untersuchungsmaterialien kostenfrei zur Verfügung gestellt haben.

\section{Interessenkonflikt}

Der korrespondierende Autor gibt an, dass kein Interessenkonflikt besteht. 


\section{References}

1. Arnold RW, Combe EC, Warford JH (2002) Bonding of stainless steel brackets to enamel with a new self-etching primer. Am J Orthod Dentofacial Orthop 122:274-276

2. Artun J, Bergland S (1984) Clinical trials with crystal growth conditioning as an alternative to acid-etch enamel pretreatment. Am J Orthod 85:333-340

3. Attar N, Taner TU, Tülümen E, Korkmaz Y (2007) Shear bond strength of orthodontic brackets bonded using conventional vs one and two step self-etching/ adhesive systems. Angle Orthod 77:518-523

4. Bishara SE, Ajlouni R, Laffoon JF (2003) Effect of thermocycling on the shear bond strength of a cyanoacrylate orthodontic adhesive. Am J Orthod Dentofacial Orthop 123:21-24

5. Bishara SE, Ajlouni R, Laffoon JF, Warren JJ (2006) Comparison of shear bond strength of two self-etch primer/adhesive systems. Angle Orthod 76:123-126

6. Bishara SE, Otsby AW, Ajlouni R et al (2008) A new premixed self-etch adhesive for bonding orthodontic brackets. Angle Orthod 78:1101-1104

7. Buonocore MG (1955) A simple method of increasing the adhesion of acrylic filling materials to enamel surfaces. J Dent Res 34:849-853

8. Buyukyilmaz T, Usumez S, Karaman Al (2003) Effects of self-etching primers on bond strength-are they reliable? Angle Orthod 73:64-70

9. Cal-Neto JP, Quintao CA, Oliveira Almelda MA, Miguel JAM (2009) Bond failure rates with a self-etching primer: a randomized controlled trial. Am J Orthod Dentofacial Orthop 135:782-786

10. Cehreli ZC, Kecik D, Kocadereli I (2005) Effect of self-etching primer and adhesive formulations on the shear bond strength of orthodontic brackets. Am J Orthod Dentofacial Orthop 127:573-579

11. Cehreli ZC, Stephan A, Sener B (2003) Antimicrobial properties of self-etching primer-bonding systems. Oper Dent 28:143-148

12. Cheong C, King NM, Pashley DH et al (2003) Incompatibility of self-etching adhesives with chemical/dual cured composites: two-step vs. one-step systems. Oper Dent 28:747-755

13. Cinader D (2001) Chemical processes and performance comparisons of Transbond Plus self-etching primer. Orthod perspect 8:5-6

14. Elekdag-Turk S, Cakmak F, Isci D, Turk T (2008) 12-Month self-ligating bracket failure rate with a self-etching primer. Angle Orthod 78:1095-1100

15. Elekdag-Turk S, Isci D, Turk T, Cakmak F (2008) Six-month bracket failure rate evaluation of a self-etching primer. Eur J Orthod 30:211-216

16. Elekdag-Turk S, Turk T, Isci D, Ozkalyci N (2008) Thermocycling effects on shear bond strength of a self-etching primer. Angle Orthod 78:351-356

17. Eminkahyagil N, Korkmaz Y, Gokalp S, Baseren M (2005) Shear bond strength of orthodontic brackets with newly developed antibacterial self-etch adhesive. Angle Orthod 75:843-848

18. Faltermeier A, Behr M, Müßig D (2007) A comparative evaluation of bracket bonding with 1-, 2- and 3-component adhesive systems. Am J Orthod Dentofacial Orthop 132:144.e1-5

19. Ferracane JL, Berge HX, Condon JR (1998) In vitro aging of dental composites in water-effect of degree of conversion, filler volume and filler/matrix coupling. J Biomed Mater Res 42:465-472

20. Fjeld M, Ogaard B (2006) Scanning electron microscopic evaluation of enamel surfaces exposed to 3 orthodontic bonding systems. Am J Orthod Dentofacial Orthop 130:575-581

21. Fritz UB, Diedrich P, Finger WJ (2001) Self-etching primers - an alternative to the conventional acid etch technique? J Orofac Orthop 3:238-245

22. Galan D, Williams PT, Kasloff Z (1991) Effects of warm air-drying and spreading on resin bonding. Am J Dent 4:277-280

23. Glasspoole EA, Erickson RL, Davidson CL (2001) Effect of enamel pretreatments on bond strength of compomer. Dent Mater 17:402-408

24. Gwinnett AJ, Yu S (1995) Effect of long-term water storage on dentin bonding. Am J Dent 8:109-111

25. Imazato S, Kuramoto A, Kaneko T et al (2002) Comparison of antibacterial activity of simplified adhesive systems. Am J Dent 15:356-360

26. Ireland AJ, Knight H, Sherriff M (2003) An in vivo investigation into bond failure rates with a new self-etching primer system. Am J Orthod Dentofacial Orthop 124:323-326

27. Kanemura N, Sano H, Tagami J (1999) Tensile bond strength and SEM evaluation of ground and intact enamel surfaces. J Dent 27:523-530

28. Kitsako Y, Burrow MF, Nikaido T, Tagami J (2000) The influence of storage solution on dentin bond durability of resin cement. J Dent 16:1-6
29. Kugel G, Ferrari M (2000) The science of bonding: from first to sixth generation. J Am Dent Assoc 13[Suppl]:20S-25S

30. Murfitt PG, Quick AN, Swain MV, Herbison GP (2006) A randomised clinical trial to investigate bond failure rates using a self-etching primer. Eur J Orthod 28:444-449

31. Murray SD, Hobson RS (2003) Comparison of in vivo and in vitro shear bond strength. Am J Orthod Dentofacial Orthop 123:2-9

32. Nathanson D, Bodkin JL, Evans JR (1982) SEM of etching patterns in surface and subsurface enamel. J Pedod 7:11-17

33. Newman GV (1965) Epoxy adhesives for orthodontic attachments: progress report. Am J Orthod 51:901-912

34. Ostby AW, Bishara SE, Denehy GE et al (2008) Effect of self-etchant pH on the shear bond strength of orthodontic brackets. Am J Orthod Dentofacial Orthop 134:203-208

35. Pandis N, Eliades T (2005) A comparative in vivo assessment of the long-term failure rate of 2 self-etching primers. Am J Orthod Dentofacial Orthop 128:9698

36. Paschos E, Kurochkina N, Huth KC et al (2009) Failure rate of brackets bonded with antimicrobial and fluoride-releasing, self-etching primer and the effect on prevention of enamel demineralization. Am J Orthod Dentofacial Orthop 135:613-620

37. Pashley DH, Tay FR (2001) Aggressiveness of contemporary self-etching adhesives. Part II: Etching effects on unground enamel. Dent Mater 17:430-444

38. Pasquale A, Weinstein M, Borislow AJ, Braitman LE (2007) In-vivo prospective comparison of bond failure rates of 2 self-etching primer/adhesive systems. Am J Orthod Dentofacial Orthop 671-674

39. Retief DH (1974) Failure at the dentin adhesive-etched enamel interface. J Oral Rehabil 1:265-284

40. Ripa LW, Gwinnet AJ, Buonocore MG (1966) The 'prismless' outer layer of deciduous and permanent enamel. Arch Oral Biol 11:41-48

41. Sanares AM, Itthagarun A, King NM et al (2001) Adverse surface interactions between one-bottle light-cured adhesives and chemical-cured composites. Dent Mater 17:542-556

42. Scougall-Vilchis RJ, Ohashi SH, Yamamoto K (2009) Effects of 6 self-etching primers on shear bond strength of orthodontic brackets. Am J Orthod Dentofacial Orthop 135:424e1-7

43. Scougall-Vilchis RJ, Yamamoto S, Kitai N, Yamamoto K (2009) Shear bond strength of orthodontic brackets bonded with different self-etching adhesives. Am J Orthod Dentofacial Orthop 136:425-430

44. Shinchi MJ, Soma K, Nakabayashi N (2000) The effect of phosphoric acid concentration on resin tag length and bond strength of a photo-cured resin to acidetched enamel. Dent Mater 16:324-329

45. Sturdevant CM, Barton RE, Sockwell CL, Strickland WD (1985) The art and science of operative dentistry, 2nd edn. W.B. Saunders, Philadelphia, pp 54-55

46. Tay FR, Pashley DH, Carvalho RM, Itthagarun A (2002) Single-step adhesives are permeable membranes. J Dent 30:371-382

47. Tay FR, Pashley DH, Suh B et al (2004) Single-step, self-etch adhesives behave as permeable membranes after polymerization. Part I. Bond strength and morphologic evidence. Am J Dent 17:271-278

48. Van Landuyt KL, Yoshida Y, Hirata I et al (2008) Influence of the chemical structure of functional monomers on their adhesive performance. J Dent Res 87:757761

49. Velo S, Carano A (2002) Self-etching versus traditional bonding systems in orthodontics: an in vitro study. Orthod Craniofac Res 5:166-169

50 Whittaker DK (1982) Structural variations in the surface zone of human tooth enamel observed by scanning electron microscopy. Arch Oral Biol 27:383-392

\section{Correspondence address}

Dr. Dr. Lorenz Brauchli

Klinik für Kieferorthopädie und Kinderzahnmedizin

Hebelstrasse 3

4056 Basel

Switzerland

e-mail:Lorenz.Brauchli@unibas.ch 